\title{
Improvement of the nutraceutical quality of broccoli sprouts by elicitation
}

\author{
Fausta Natella ${ }^{\mathrm{a}, *}$, Mariateresa Maldini $^{\mathrm{b}}$, Mirella Nardini ${ }^{\mathrm{a}}$, Elena Azzini ${ }^{\mathrm{a}}$, Maria Stella Foddai ${ }^{\mathrm{a}}$, \\ Anna Maria Giusti ${ }^{c}$, Simona Baima ${ }^{a}$, Giorgio Morelli ${ }^{a}$, Cristina Scaccini ${ }^{\mathrm{a}}$ \\ ${ }^{a}$ CREA-NUT, Consiglio per la Ricerca in Agricoltura e l'Analisi dell'Economia Agraria, Food and Nutrition Research Centre, Via Ardeatina 546, 00178 Roma, Italy \\ ${ }^{\mathrm{b}}$ Chemistry and Pharmacy Department, University of Sassari, via Muroni 23, 07100 Sassari, Italy

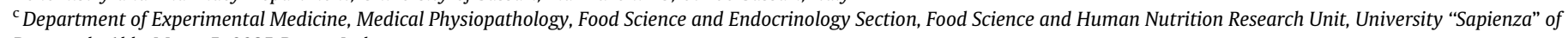 \\ Rome, p.le Aldo Moro, 5, 0085 Roma, Italy
}

\section{A R T I C L E I N F O}

\section{Article history:}

Received 2 September 2015

Received in revised form 3 December 2015

Accepted 15 January 2016

Available online 20 January 2016

\section{Keywords:}

Broccoli sprouts

Bioactive molecules

Glucosinolate

Phenolic compound

Anthocyanin

Elicitor

\begin{abstract}
A B S T R A C T
Epidemiological studies show an inverse association between Brassica consumption and chronic diseases. Phytochemicals are thought to be beneficial for human health and therefore responsible for this protective effect. Increasing their levels into Brassica food is considered an expedient nutritional strategy that can be achieved through the manipulation of growth conditions by elicitors. In this work we systematically evaluated the influence of treatment with different elicitors (sucrose, mannitol, $\mathrm{NaCl}$, 1-aminocyclopropane-l-carboxylic acid, salicylic acid and methyl jasmonate) on the phytochemical composition of broccoli sprouts. The content of total and single glucosinolates, total phenolic compounds, total flavonoids, total anthocyanins, vitamin $\mathrm{C}$ and $\mathrm{E}$ and $\beta$-carotene was assessed.

The exposure to different elicitors produced concentration- and elicitor-dependent specific changes in the content of all the phytochemicals considered. Sucrose, identified as the most effective elicitor by principal component analysis, induced a significant increase of total and specific glucosinolates, vitamin C, total anthocyanins and polyphenols. Sucrose is likely to represent an effective tool to increase the nutritional value of broccoli sprouts.
\end{abstract}

(c) 2016 Elsevier Ltd. All rights reserved.

\section{Introduction}

Epidemiological evidence suggests that there is an inverse association between consumption of cruciferous vegetables (such as broccoli, brussel sprouts and cauliflower) and the risk of many types of cancer (Liu \& Lv, 2013) and cardiovascular disease (Cornelis, El-Sohemy, \& Campos, 2007). These vegetables contain numerous bioactive compounds (such as vitamins, minerals, glu-

Abbreviations: ANOVA, one-way analysis of variance; ACC, 1aminocyclopropane-l-carboxylic acid; ESI-MS, electrospray ionization sourcemass spectrometry; FRAP, ferric reducing antioxidant power; GAE, gallic acid equivalents; GL, glucosinolate; HPLC, high performance liquid chromatography; ITC, isothiocyanates; MeJa, methyl jasmonate; PCA, principal component analysis; SA, salicylic acid; THF, tetrahydrofuran; TEAC, trolox equivalent antioxidant capacity.

* Corresponding author.

E-mail addresses: fausta.natella@entecra.it (F. Natella), mtmaldini@uniss.it (M. Maldini), mirella.nardini@entecra.it (M. Nardini), elena.azzini@entecra.it (E. Azzini), mariastella.foddai@entecra.it (M.S. Foddai), annamaria.giusti@ uniroma1.it (A.M. Giusti), simona.baima@entecra.it (S. Baima), Giorgio.morelli@ entecra.it (G. Morelli), cristina.scaccini@entecra.it (C. Scaccini). cosinolates and phenolic compounds) that are considered to be responsible for their health-promoting properties.

In particular, glucosinolates (GL), and their hydrolysis products isothiocyanates (ITC), are considered to have an important role in cancer chemoprevention. These compounds, in fact, are reported to have a chemo-preventative activity in a variety of cell and animal models, probably due to their capacity to induce phase 2 detoxification enzymes (Plumb et al., 1996). In addition, cruciferous vegetables are an excellent dietary source of other health promoting phytochemicals, such as vitamins ( $C$ and $E$ ), carotenoids and phenolic compounds (among them anthocyanins), having an established role in the prevention of several chronic diseases (Björkman et al., 2011).

According to these evidences, many studies have focused on strategies aimed to increase the content of bioactive compounds in cruciferous vegetables. Recently, young seedlings (sprouts) have attracted an increasing interest by nutritionists, as they possess a higher nutritional value than adult plants. Broccoli sprouts, in fact, have higher level of GLs (Pérez-Balibrea, Moreno, \& García-Viguera, 2011), and phenolic compounds (Singh, Upadhyay, Prasad, 
Bahadur, \& Rai, 2007), and a higher phase 2 inducing activity (Fahey, Zhang, \& Talalay, 1997) than adult plants. Sprouts can be easily grown at home and, irrespective of the season, they only need water and light for growth. Sprouts can be consumed raw, thus avoiding the unavoidable loss of bioactive molecules during cooking and/or processing. Therefore, sprouts could be used as a natural functional food.

Obviously, environmental growth conditions affect the synthesis and therefore the content of many of the health promoting bioactive molecules present in plants. Abiotic (temperature, light, radiation, water, exposure to salt or metals) and biotic (herbivory, fungal, bacterial and/or viral infection) stresses can affect both the content and profile of these phytochemicals (Björkman et al., 2011). Also, broccoli sprout composition is affected by environmental conditions and it has been demonstrated that light or sucrose treatment increase phytochemical composition in terms of vitamins, GLs and phenolic compounds (Guo, Yuan, \& Wang, 2011b; Pérez-Balibrea et al., 2011).

To our knowledge, however, data currently available are scarce and fragmented. In fact, previous studies evaluated the effect of a limited number of inducers on few bioactive molecules, not considering the overall range of phytochemicals. Moreover, these studies are hardly comparable due to the wide different growth conditions taken in consideration (e.g. seedling age, treatment period).

The aim of this study was to systematically evaluate, in broccoli sprouts, the influence of different concentrations of many different elicitors (sucrose, mannitol, $\mathrm{NaCl}, 1$-aminocyclopropane-1carboxylic acid (ACC), salicylic acid (SA) and methyl jasmonate (Meja)) on the content of many different bioactive molecules (total and individual glucosinolates, total phenolic compounds, total flavonoids, total anthocyanins, vitamin $C$ and $E$ and $\beta$-carotene). Moreover, taking into account the complexity of the interaction among these phytochemicals, we utilized principal component analysis (PCA) to assess the conditions (type of elicitor and dose) able to provide the most significant overall effect on phytochemical composition of broccoli sprouts.

\section{Materials and methods}

\subsection{Materials}

Solvents and HPLC grade methanol used for extraction were of high purity (Carlo Erba, Milano, Italy). Acetonitrile and formic acid were from Sigma-Aldrich Chemical Company (St Louis, MO). HPLC grade water $(18 \mathrm{~m} \Omega$ ) was prepared using a Millipore (Bedford, MA, USA) Milli-Q purification system. Glucobrassicin potassium salt, glucoraphanin potassium salt, glucoiberin potassium salt, glucoerucin potassium salt, progoitrin potassium salt, gluconapin potassium salt, sinigrin potassium salt and glucocheirolin potassium salt were purchased from PhytoLab GmbH \& Co. KG (Vestenbergsgreuth, Germany). Folin \& Ciocalteau's phenol reagent, gallic acid, $(+)$ catechin, aluminium chloride, sodium nitrite were from Sigma-Aldrich (St. Louis, MO, USA).

\subsection{Growth condition of broccoli sprouts}

Broccoli seeds (Brassica oleracea L. var. botrytis subvar. cymosa) were purchased from SUBA\&UNICO (Longiano, FC, Italy). Seeds were surface sterilized by incubating for $15 \mathrm{~min}$ in $40 \%$ bleach ( $2 \%$ sodium hypochlorite) with shaking, than drained and rinsed 10 times with distilled water. After soaking, in distilled water, for 16-18 h (overnight) at $21^{\circ} \mathrm{C}$, seeds were rinsed in distilled water and transferred in the germination cylinder, Vitaseed sprouter (SUBA\&UNICO, Longiano, FC, Italy). Sprouts were grown at $21^{\circ} \mathrm{C}$ in a plant growth chamber (Weiss Gallenkamp, Loughborough, United Kingdom) equipped with PHILIPS Master TL-D 36W/840 cool-white fluorescent tubes providing a photosynthetic photon flux density of $110 \mathrm{mmol} \mathrm{m}^{-2} \mathrm{~s}^{-1}$, with a $16 \mathrm{~h} \mathrm{light} / 8 \mathrm{~h}$ dark photoperiod and subjected to different stressors.

Mannitol, sucrose, $\mathrm{NaCl}, \mathrm{ACC}, \mathrm{SA}$ and Meja were selected as elicitors, for their known activity as stress inductors or signal molecules: mannitol is an inductor of osmotic stress, $\mathrm{NaCl}$ is an inductor of salt stress, SA is a plant hormone regulating resistance to fungal, bacteria and viral pathogen, ACC is a precursor of ethylene and a signal molecule, Meja is a plant hormone regulating resistance against insect, and sucrose is a carbon source, an inductor of osmotic stress and a modulator of developmental and metabolic process (Baenas, García-Viguera, \& Moreno, 2014a). Each elicitor was used at different concentrations that were selected according to previous studies. Mannitol and sucrose were dissolved in water at 88 and $176 \mathrm{mM}$ concentrations. $\mathrm{NaCl}$ was dissolved in water at 10,50 and $100 \mathrm{mM}$ concentrations. SA was dissolved in water at 250 and $750 \mu \mathrm{M}$ concentrations. ACC was dissolved in ethanol and added at 50 and $500 \mu \mathrm{M}$ final concentrations. MeJa was dissolved in ethanol and added at 30 and $300 \mu \mathrm{M}$ final concentrations. All solutions were freshly prepared before application and used to replace water in the sprouter 3 days after sowing. The sprouts were grown in the presence of the elicitors for 2 more days, for a total of 5-day germination. This time point was selected as five day-old sprouts still possess both a suitable biomass and a high phytochemical content; it is in fact known that, in the course of the germination, there is a steady decline of the major bioactive molecules (Maldini, Baima, Morelli, Scaccini, \& Natella, 2012). Treated and control 5-day-old sprouts samples were rapidly and gently collected from the surface of the germination cylinder at midday, weighed (fresh mass) and immediately frozen in liquid nitrogen and stored at $-80^{\circ} \mathrm{C}$ for further analysis. Frozen sprouts were ground to a fine powder in a Waring blender, cooled with liquid nitrogen and aliquots of sprouts powder were used for humidity content determination and further analysis.

\subsection{GLs determination}

For GLs measure, each sample of broccoli sprouts was extracted with methanol:water $(70: 30 \mathrm{v} / \mathrm{v}$; sample to solvent ratio $1: 25 \mathrm{w} / \mathrm{v})$ at $70{ }^{\circ} \mathrm{C}$ for $30 \mathrm{~min}$ under vortex mixing to facilitate the extraction. The samples were successively centrifuged $(4000 \mathrm{rpm}, 30 \mathrm{~min}$, $4{ }^{\circ} \mathrm{C}$ ), the supernatants were collected and the solvent was completely removed, using a rotary evaporato, under vacuum at $40{ }^{\circ} \mathrm{C}$. The dried samples were dissolved in ultrapure water with the same volume of extraction and filtered through $0.20 \mathrm{~mm}$ syringe PVDF filters (Whatmann International Ltd., UK).

GLs analyses were performed as previously described (Maldini et al., 2012), using an HPLC system (Perkin-Elmer, USA) interfaced to an Applied Biosystems (Foster City, CA, USA) API3200 Q-Trap spectrometer and GLs were analyzed. Briefly, quantitative on-line HPLC-ESI-MS/MS analyses were performed using the mass spectrometer in MRM mode. Samples were injected $(10 \mu \mathrm{l})$ into a Luna $\mathrm{C}_{18}$ column (Phenomenex, USA) $(150 \times 2.1 \mathrm{~mm}$ i.d., $5 \mu \mathrm{m} \mathrm{d})$ and eluted at flow rate of $0.3 \mathrm{ml} \mathrm{min}{ }^{-1}$. Mobile phase $A$ was $\mathrm{H}_{2} \mathrm{O}$ containing $0.1 \%$ formic acid while mobile phase $B$ was acetonitrile containing $0.1 \%$ formic acid. Elution gradient was: $0^{\prime} 6 \% \mathrm{~B}, 0.1-$ $15 \mathrm{~min} \rightarrow 12 \% \quad \mathrm{~B}, \quad 15.1-21 \mathrm{~min} \rightarrow 25 \% \quad \mathrm{~B}, \quad 21.1-30 \mathrm{~min} \rightarrow 60 \% \mathrm{~B}$, $30.1-31 \mathrm{~min} \rightarrow 100 \% \mathrm{~B}, 31.1-38 \mathrm{~min} 100 \% \mathrm{~B}$ and finally for reequilibration: $38.1-39$ min $\rightarrow 6 \% \mathrm{~B}, 39.1-45$ min $6 \% \mathrm{~B}$. The column was kept at $25^{\circ} \mathrm{C}$, using a Peltier Column Oven Series 200 (Perkin Elmer). The source temperature was held at $450^{\circ} \mathrm{C}$, and MS parameters were those optimised for the ESI-MS and ESI-MS/MS analyses with ion spray voltage at $-4300 \mathrm{~V}$. For all compounds, dwell time 
was $60 \mathrm{~ms}$ and the voltage applied was $-4500 \mathrm{~V}$. Data acquisition and processing were performed using Analyst software 1.5.1.

\subsection{Total ascorbic acid determination}

For ascorbic acid measurements, each sample of broccoli sprouts was extracted following the method described by Margolis et al. (Margolis, Paule, \& Ziegler, 1990). The quantitative analyses were performed by an HPLC system equipped with a coulometric detector (ESA model 580, Chemsford, MA, USA). The setting potential was $0,100,200,300$ and $400 \mathrm{mV}$ (v. Palladium reference electrode).

The chromatographic separation was obtained using a $250 \times 4.6$ mm Capcell Pak NH 2 column (Shiseido, Tokyo, Japan). The column was equilibrated at $40^{\circ} \mathrm{C}$ at a flow-rate of $0.8 \mathrm{ml} /$ min with a solvent composed of $0.680 \mathrm{~g}$ monobasic potassium phosphate, $200 \mathrm{ml}$ water, $800 \mathrm{ml}$ acetonitrile and $7.5 \mathrm{ml}$ concentrated phosphoric acid.

\section{5. $\alpha$-Tocopherol and $\beta$-carotene determination}

Vitamin $\mathrm{E}$ and $\beta$-carotene were extracted following the method described by Sharpless et al. (Sharpless, Arce-Usuna, Thomas, \& Gill, 1999). The sample was saponified in methanol/tetrahydrofuran (THF), then the reaction mixture was extracted with hexane/ diethylether, the extract was evaporated and the residue was dissolved in hexane. The extract in hexane was evaporated and reconstituted in a mobile phase of methanol/acetonitrile/THF 50:45:5, then analysed by HPLC according to Maiani et al. (Maiani et al., 1995). The chromatographic separation was obtained using a Waters Nova Pak C18, $3.9 \mathrm{~mm} \times 150 \mathrm{~mm}$ column (Waters Corporation, Milford, Massachusetts, USA).

\subsection{Total anthocyanins determination}

Anthocyanins were determined by spectrophotometric method according to Abdel-Aal and Huel, (Abdel-Aal \& Hucl, 1999). A ground broccoli sprouts sample $(1 \mathrm{~g})$ was weighed in a $50 \mathrm{ml}$ centrifuge tube, and $8 \mathrm{ml}$ of acidified ethanol (ethanol and $\mathrm{HCl} 1.0 \mathrm{~N}$, $85: 15, \mathrm{v} / \mathrm{v}$ ) was added. The solution was mixed and adjusted to $\mathrm{pH}=1$ with $4 \mathrm{~N} \mathrm{HCl}$. The resulting solution was shaken for $30 \mathrm{~min}$ in the dark. The tube was centrifuged at $4000 \mathrm{rpm}$ for $30 \mathrm{~min}$ at $5{ }^{\circ} \mathrm{C}$ and the supernatant was collected, placed into a $50-\mathrm{ml}$ volumetric flask and brought to volume $(16,67 \mathrm{ml})$ with acidified ethanol. Absorbance was measured at $535 \mathrm{~nm}$ against a blank. Cyanidin 3-glucoside or kuromanin (Sigma) was used as standard.

Total anthocyanin content per sample $(\mathrm{mg} / \mathrm{kg})$ was calculated as cyaniding 3-glucoside following formula:

$C=(A / \varepsilon) \times(\operatorname{vol} / 1000) \times \mathrm{MW} \times(1 /$ sample $\mathrm{wt}) \times 10^{6}$

where $C$ is concentration of total anthocyanins $(\mathrm{mg} / \mathrm{kg}), A$ is the absorbance reading, $\varepsilon$ is molar absorptivity (cyaniding 3-glucoside $=25,965 \mathrm{~cm}^{-1} \mathrm{M}^{-1}$ ), vol is total volume of anthocyanins extract, and MW is molecular weight of cyanidin 3-glucoside $=484,82$.

\subsection{Total polyphenol and total flavonoid determination}

For polyphenols and flavonoid measure, frozen sprouts (200 mg) were extracted with $2 \mathrm{ml}$ absolute methanol for $15 \mathrm{~min}$ at room temperature. The supernatant was collected after centrifugation ( $2000 \mathrm{~g}$ for $5 \mathrm{~min}$ ). The procedure was repeated twice. For each sample, supernatants were combined, stored at $-20{ }^{\circ} \mathrm{C}$ and used for analyses within $72 \mathrm{~h}$.
The total polyphenols content of extracts was determined by the Folin-Ciocalteu's method (Kapasakalidis, Rastall, \& Gordon, 2006; Singleton, Orthofer, \& Lamuela-Raventós, 1999). Gallic acid was used as a reference compound for the calibration curve in the range of $1-20 \mu \mathrm{g}$. All measurements were performed in triplicate. Absorbance values of samples were converted to gallic acid equivalents (GAE).

The total flavonoids content of extracts was determined by using a colorimetric method described previously (Dewanto, $\mathrm{Wu}$, Adom, \& Liu, 2002). (+) Catechin was used as reference compound for the calibration curve in the range of $2.5-50 \mu \mathrm{g}$. All measurements were performed in triplicate. Absorbance values of samples were converted to catechin equivalents.

We tested both sucrose and mannitol for possible interferences on the FC and flavonoid assays and observed no interferences at all at least up to $10 \mathrm{mg} / \mathrm{ml}$, which was the highest concentration tested.

\subsection{Total antioxidant capacity}

Total antioxidant capacity was measured using two different assays: ferric reducing antioxidant power (FRAP) (Benzie \& Strain, 1996) and Trolox equivalent antioxidant capacity (TEAC) method (Pellegrini et al., 2003).

\subsection{Data analysis and statistics}

Data were expressed as mean \pm SD and analysed by one way ANOVA followed by Tukey's test. Differences versus control were considered significant at a value of $p<0.05$.

For principal component analysis (PCA) data were normalised by centering and autoscaling; the results of the analysis are presented in term of score and loading plots.

Data analysis and statistics were performed using XLSTAT software (Addinsoft, 2010).

\section{Results}

\subsection{Glucosinolates}

Total GLs and individual GLs were quantitatively determined in broccoli sprouts. Fourteen GLs were detected, namely: 4 aliphatic (gluconapin, progoitrin/epiprogoitrin, sinigrin and gluconapoleiferin), 6 aliphatic with an additional sulphur atom (glucoraphanin, glucoiberin, glucoerucin glucocheirolin, glucoiberverin and glucoalysin) and 4 indolic (4-methoxyglucobrassicin, neoglucobrassicin, 4-hydroxyglucobrassicin and glucobrassicin).

The amount of total GLs was strongly and differently affected by the different treatments. Compared to the control, mannitol $176 \mathrm{mM}$ and sucrose both 176 and $88 \mathrm{mM}$ induced a significant increase of total GLs concentration (Fig. 1), with an increase of $81 \%, 82 \%$ and $73 \%$ compared to control, respectively. The other treatments were not associated to any statistically significant changes of total GLs content with respect to the control, even though the exposure to the lowest $\mathrm{NaCl}$ concentrations seems to induce a $-23 \%$ decrease.

Distinct effects have been observed when focusing on indolic GLs (Fig. 1). Compared to the control indolic GLs were significantly affected only by sucrose $176 \mathrm{mM}(+190 \%)$. The treatment with MeJa also induced an increase in indolic GLs but, in this case, the increase did not reach statistical significance $(p=0.136)$.

The expression of single GLs responded differently to the different stressors (Fig. 2). In comparison with the control, sucrose, mannitol and ACC seem to induce indifferently almost all the GLs, whereas SA appears to be selective for the induction of 
gluconapoleiferin, glucoalysin and 4-methoxyglucobrassicin, and MeJa for neoclucobrassicin and glucobrassicin.

\subsection{Polyphenols}

Total polyphenols, total flavonoids and total anthocyanins in broccoli sprouts, grown in different conditions, are shown in Fig. 3(A, B, C).

In comparison with the control, total polyphenols levels were significantly increased by mannitol (both 88 and $176 \mathrm{mM}$ ), sucrose both 88 and $176 \mathrm{mM}, \mathrm{NaCl} 100 \mathrm{mM}$, ACC $500 \mu \mathrm{M}$ and SA $750 \mu \mathrm{M}$ (Fig. $3 \mathrm{~A}$ ). The percentage of increase ranged from $+12 \%$ to $+63 \%$, after the treatment with $\mathrm{NaCl} 100 \mathrm{mM}$ and sucrose $176 \mathrm{mM}$, respectively.

Similarly, the total flavonoid concentration was significantly increased by mannitol, both 88 and $176 \mathrm{mM}$, sucrose, both 88 and $176 \mathrm{mM}, \mathrm{ACC}$, both 250 and $500 \mu \mathrm{M}$, and SA, both 250 and $750 \mu \mathrm{M}$ (Fig. 3B). In this case, however, the greatest increase was observed after the treatment with mannitol ( +40 and $+37 \%$ for 88 and $176 \mathrm{mM}$ mannitol, respectively).

Conversely, only sucrose, both 88 and $176 \mathrm{mM}$, and MeJa $30 \mu \mathrm{M}$ significantly affected total anthocyanin levels (Fig. 3C), with an increase of $102 \%, 136 \%$ and $55 \%$, respectively.

\subsection{Vitamin $C$ and $E$ and $\beta$-carotene}

As shown in Fig. 4A, vitamin C concentration was significantly increased only by sucrose, both 88 and $176 \mathrm{mM}$ (+77\% and $86 \%$, respectively). Other treatments did not result in any statistically significant change of concentration. However, even if not statistically significant, mannitol increased vitamin C level, while ACC, SA and MeJa induced a decrease in concentration in broccoli sprouts.

All treatments, with the exception of sucrose $88 \mathrm{mM}(+43 \%)$ and MeJa $300 \mu \mathrm{M}(-35 \%)$, did not induce any significant changes in vitamin E concentration respective to the control (Fig. 4B).
Finally, the effects of different treatments on $\beta$-carotene expression were markedly specific: ACC $500 \mu \mathrm{M}$, SA, both 250 and $750 \mu \mathrm{M}$, and MeJa $30 \mu \mathrm{M}$ induced a significant increase in $\beta$ carotene levels respect to control $(+69 \%,+36 \%,+45 \%$ and $+53 \%$, respectively) (Fig. 4C). On the contrary, treatments with mannitol $88 \mathrm{mM}$ and sucrose $176 \mathrm{mM}$ were associated with a significant reduction in $\beta$-carotene concentration $(-30 \%$ and $-61 \%$, respectively).

\subsection{Total antioxidant capacity}

When measured as TEAC (Fig. 5A), only mannitol $176 \mathrm{mM}$ treatment was able to induce a significant increase in antioxidant capacity of broccoli sprouts respective to the control. Differently when the antioxidant capacity was measured as FRAP (Fig. 5B), mannitol and SA (both concentrations) were able to induce a significant increase, while $\mathrm{NaCl}(10$ and $50 \mathrm{mM})$ and MeJa $(30 \mu \mathrm{M})$ induced a significant decrease.

\subsection{Principal component analysis (PCA)}

In order to obtain a comprehensive view, of the effect of the different treatments, on the phytochemical composition of broccoli sprouts, we utilized a PCA approach.

The computed model captured the $72 \%$ of the total observed variance with the first 2 principal components (PCs). Score and loading plots on PC2 vs. PC1 are shown in Fig. 6.

Through PCA, we could discriminate 3 groups: group 1 contained control broccoli sprouts and ACC, $\mathrm{NaCl}$ and MeJa treated broccoli sprouts, with a phytochemical composition similar or 'worse' than that of control; the second group clustered SA and mannitol treated samples and was characterized by a high antioxidant capacity; finally, group 3 was embodied sucrose treated broccoli sprouts that were characterized by an high content of GLs, anthocyanins, polyphenols and vitamin C.

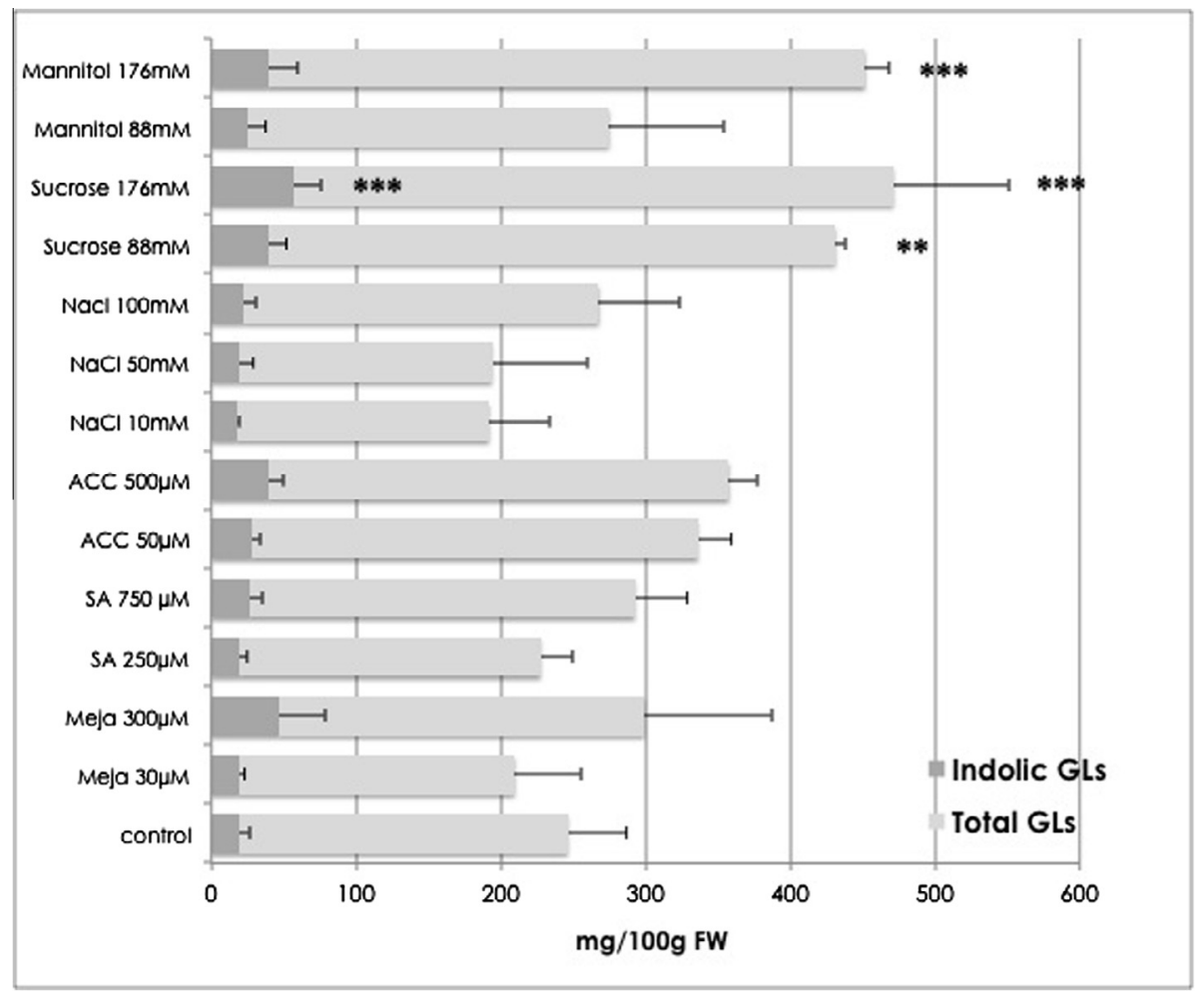

Fig. 1. Effect of elicitors on total and indolic GLs content of broccoli sprouts. 


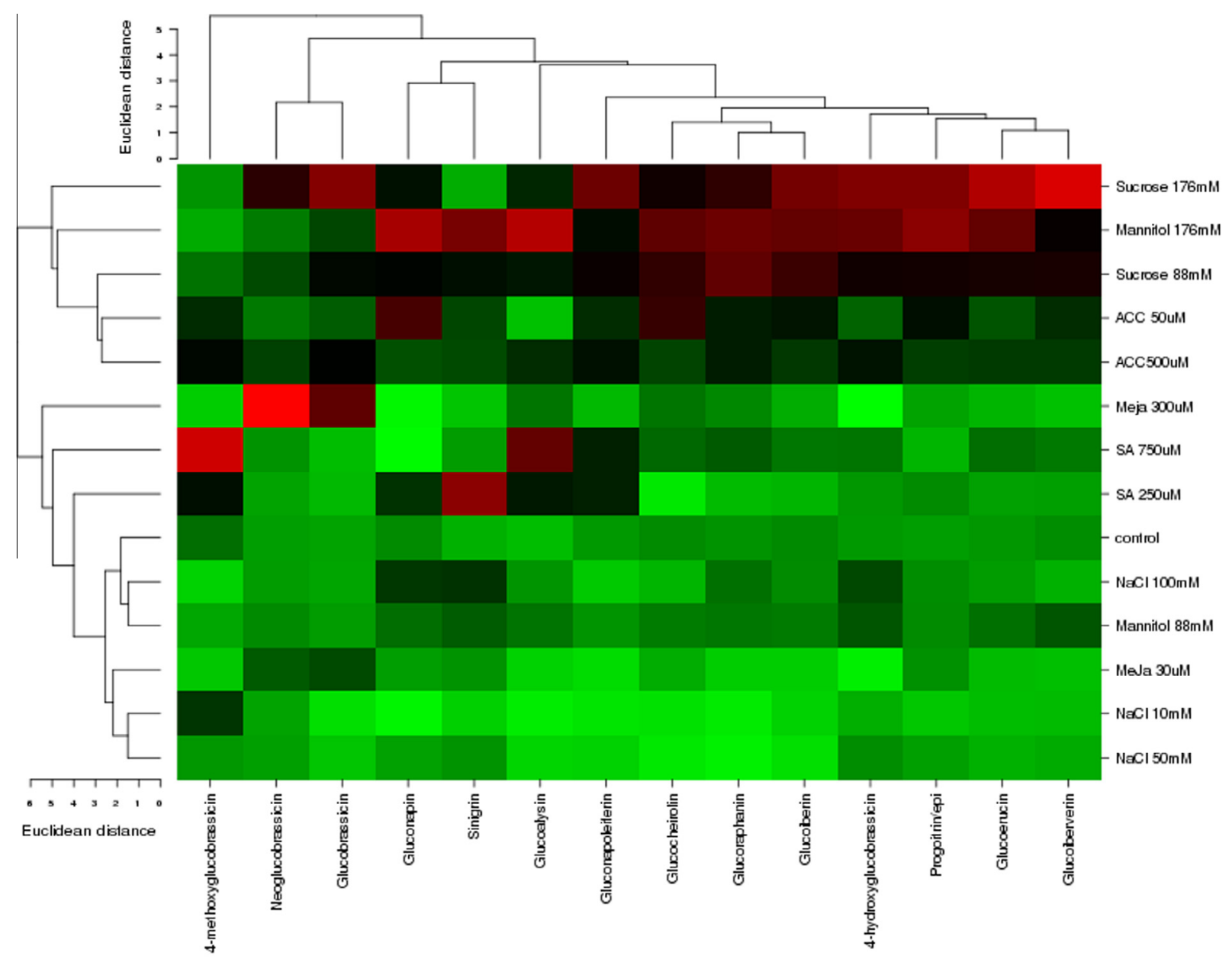

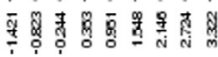

Fig. 2. Individual GLs profile of broccoli sprouts treated with different elicitors is shown through a heatmap. Mean data were normalized by $z$-score and displayed in colour scale.

A

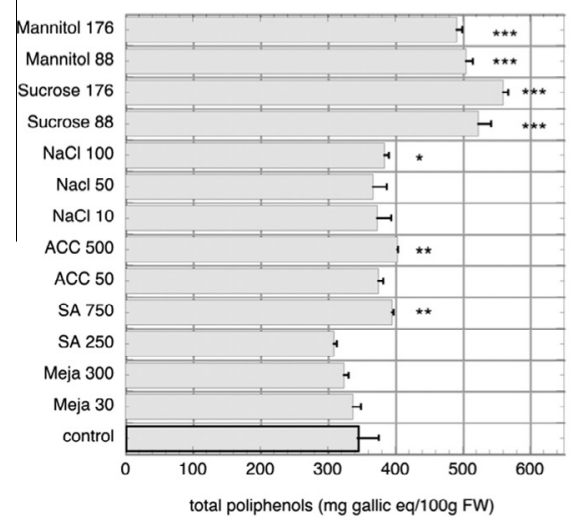

B

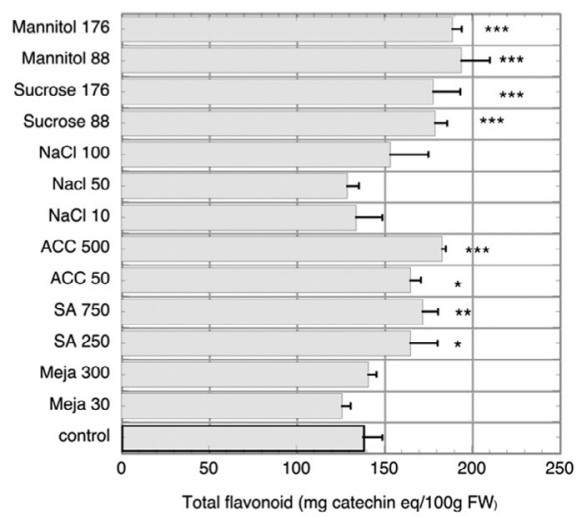

C

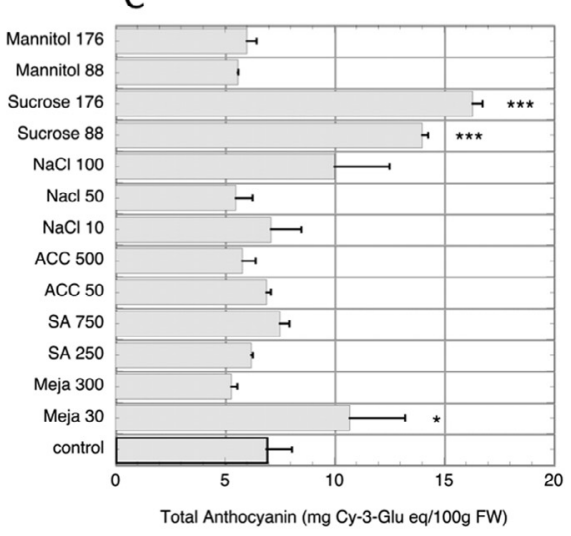

Fig. 3. Effect of elicitors on total polyphenols (A), total flavonoids (B) and total anthocyanins (C) content of broccoli sprouts.

\section{Discussion}

Secondary plant metabolites (including glucosinolates and phenolic compounds) contribute to confer to plants the capacity to adapt to their surrounding environmental changes and act as molecules involved in the plant defence system (protection from pathogens, insect, UV). In human nutrition, the same molecules represent an added value to plant-based foods, according to their 
A

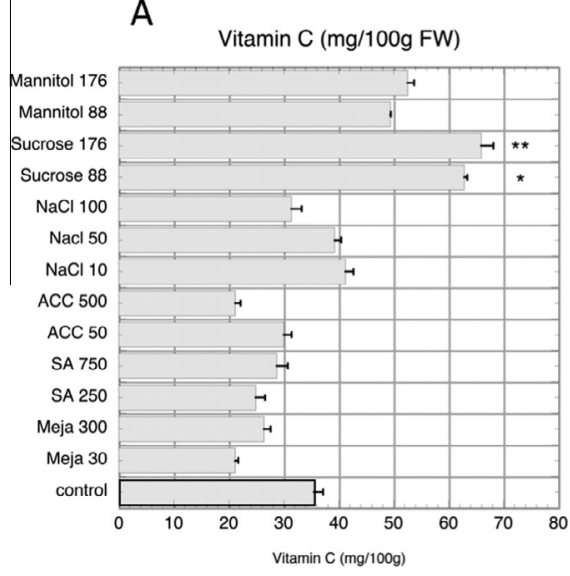

B

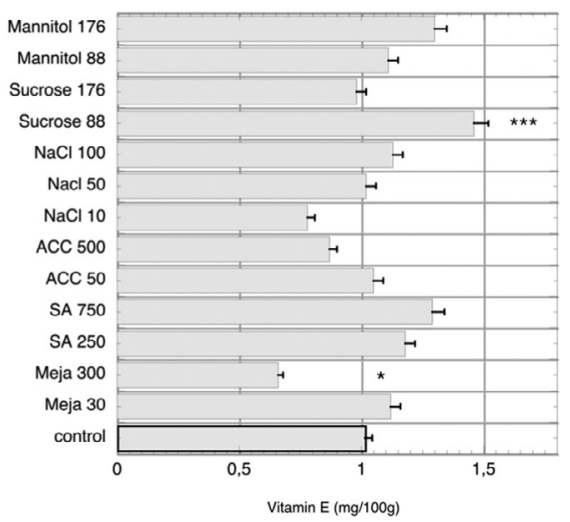

C

$\beta$-carotene (mg/100g FW)

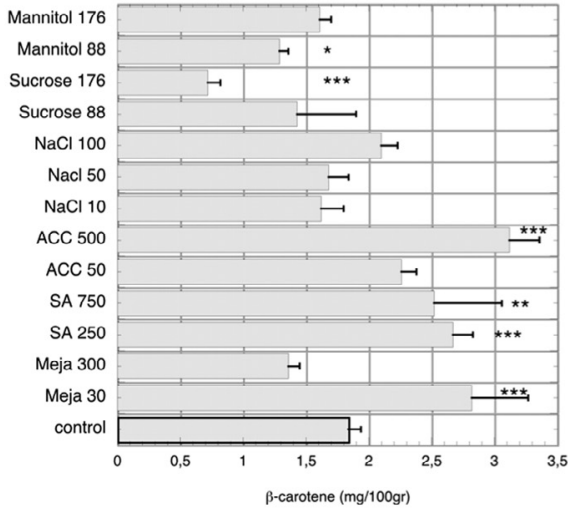

Fig. 4. Effect of elicitors on ascorbic acid (A), $\alpha$-tocopherol (B) and $\beta$-carotene (C) content of broccoli sprouts.

A

$\operatorname{TEAC}(\mathrm{mmol} \mathrm{Tx}$ eq/100g FW)

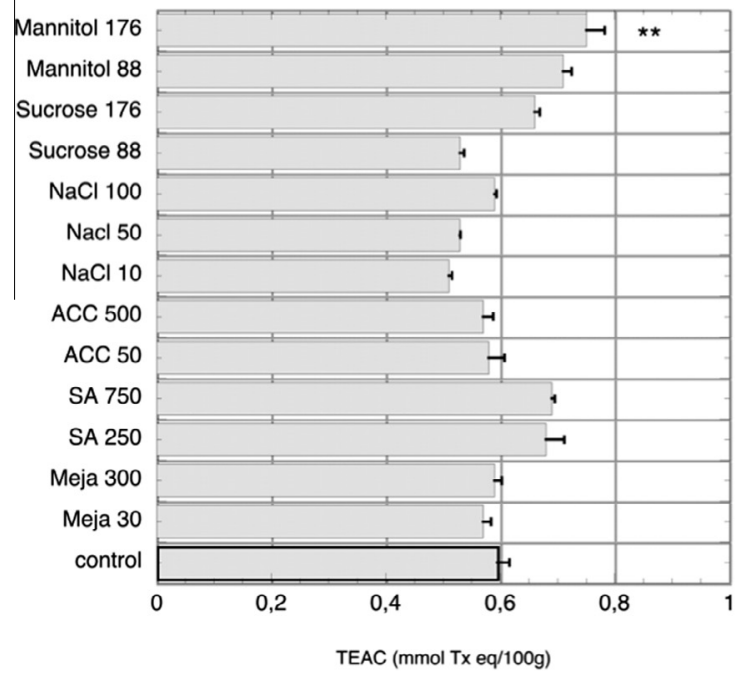

B

$\operatorname{FRAP}\left(\mathrm{mmolFe} e^{2+} / 100 \mathrm{~g} \mathrm{FW}\right)$

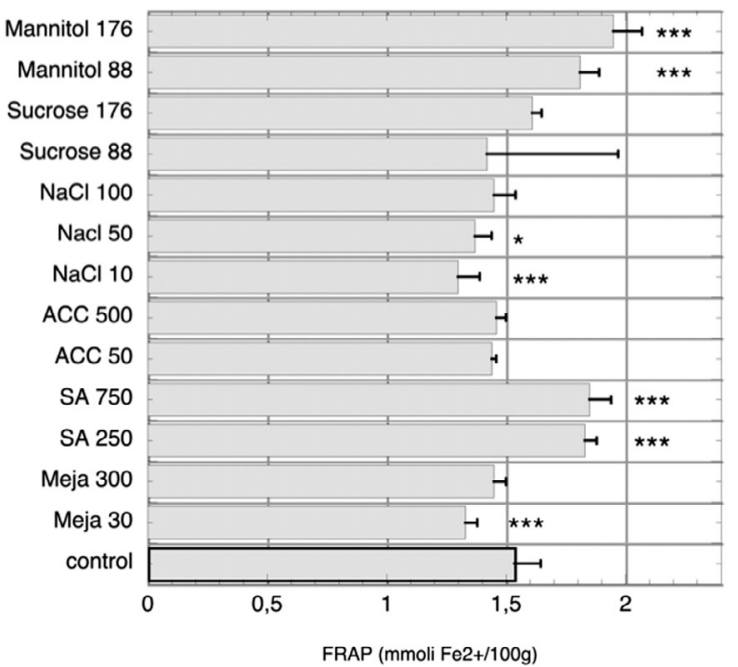

Fig. 5. Effect of elicitors on total antioxidant capacity measured as FRAP (A), and TEAC (B) content of broccoli sprouts.

health protective properties. In an attempt to improve the healthpromoting quality of vegetable foods, the administration of biotic or abiotic elicitors, that stimulate defence or stress-response in plants and the synthesis of secondary plant metabolites, is considered an expedient strategy. Elicitor nature and dose strongly affects the classes and the concentration of these secondary plant metabolites (Baenas et al., 2014a). In this study, we systematically evaluated the influence of a set of different elicitors (at different concentrations) on different classes of bioactive molecules (total and individual glucosinolates, total phenolic compounds, total flavonoids, total anthocyanins, vitamin $C$ and $E$ and $\beta$-carotene) in broccoli sprouts.

In a previous work (Maldini et al., 2012), we identified 14 different GL molecules in broccoli sprouts, including 10 aliphatic GLS (gluconapin, progoitrin/epiprogoitrin, sinigrin, gluconapoleiferin, glucoraphanin, glucoiberin, glucoerucin glucocheirolin, glucoiberverin and glucoalysin) and 4 indolic GLs (4-methoxyglucobrassicin, neoglucobrassicin, 4-hydroxyglucobrassicin and glucobrassicin). The predominant GL molecule was glucoraphanin and the total levels of GLs strongly decreased after seed germination (Maldini et al., 2012). In the present work, on the basis of the reported differential effect of different stressors on GLs expression pattern, we evaluated the effect of different elicitors on the total and individual GLs.
Previous studies showed that exogenous application of SA and MeJa could induce the accumulation of GLs in Arabidopsis (through the up-regulation of GLs biosynthetic genes) (Mikkelsen et al., 2003). Similar results were also obtained in broccoli sprouts, but only after 5 days of treatment (Baenas, García-Viguera, \& Moreno, 2014b; Pérez-Balibrea et al., 2011). In our study, SA and MeJa treatments were able to induce a slight increase of total GLs, but this increase was not statistically significant. However, by analysing single GLs we could evidence that the highest concentration of MeJa selectively induced the expression of neoglucobrassicin and glucobrassicin, two indolic GLs ( $p<0.001$ respective to the control) (data not shown). Selective and dose-dependent accumulation of indolic GLs in response to MeJa has been already reported in oilseed rape (Bodnaryk, 1994) and in broccoli florets (Ku, Jeffery, \& Juvik, 2014).

Several studies demonstrated that salt stress strongly affects GL content in different brassicaceae (López-Berenguer, MartínezBallesta, García-Viguera, \& Carvajal, 2008; Yuan, Wang, Guo, \& Wang, 2010). In radish (Yuan et al., 2010) and broccoli sprouts (Guo, Yang, Wang, Guo, \& Gu, 2014) NaCl affects GLs content in a concentration, time and cultivars dependent mode. In broccoli sprouts, an increase in glucoraphanin was observed only in some cultivar and after the application of a high $\mathrm{NaCl}$ concentration 
A

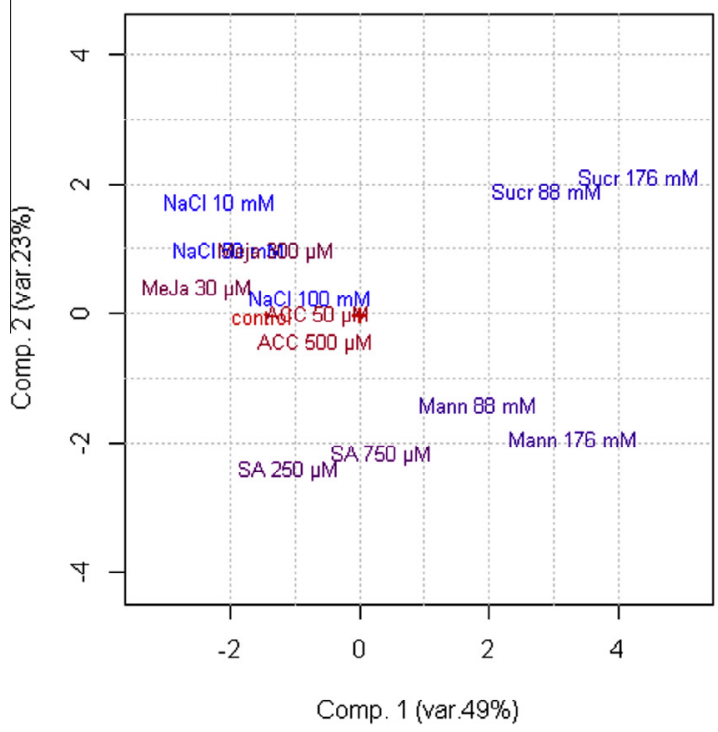

B

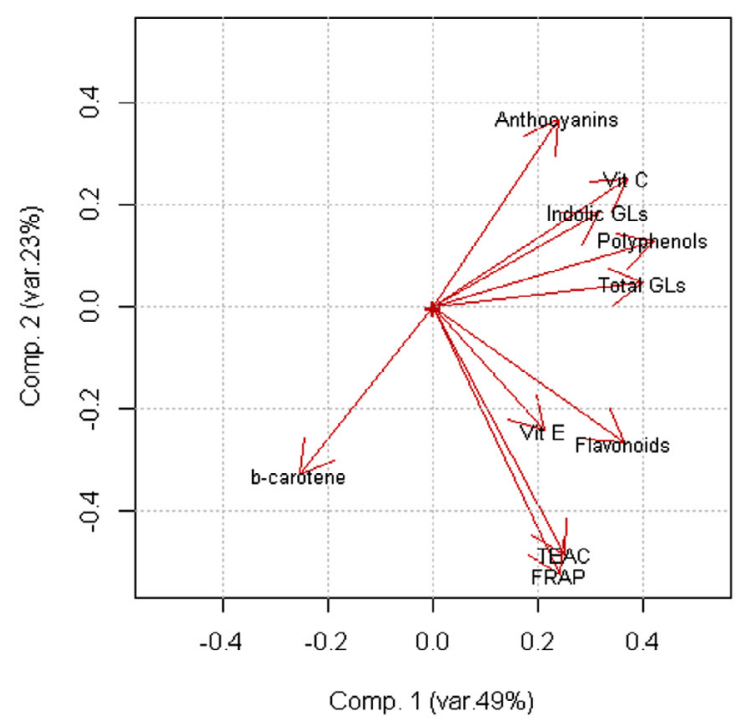

Fig. 6. Principal component analysis, (A) loadings plot and (B) score plots.

(Guo et al., 2014). In our study, even if a concentration dependent trend was observed, at all tested concentrations, $\mathrm{NaCl}$ did not significantly affect both glucoraphanin (data not shown) and total GL levels (Fig. 1).

In our study, 2-days treatment with both sucrose and mannitol enhanced total GLs level in broccoli sprouts in a concentration dependent way. Mannitol $176 \mathrm{mM}$ increased total GLs especially affecting the expression of aliphatic ones; this treatment, in fact, significantly increased glucoraphanin, glucoiberin and glucoerucin (data not shown). Conversely, the highest sucrose concentration induced both aliphatic and indolic GLs. In fact, 9 out 14 GLs (neoglucobrassicin, 4-methotyglucobrassicin, 4hydroxyglucobrassicin, glucobrassicin, glucoraphanin, glucoiberin, glucoeuricin, glucoiberverin and gluconapoleiferin) significantly increased after sucrose treatment (data not shown), indicating that sucrose-induction is less selective (Fig. 2). An increase, of total and individual GLs, has already been observed using different sugars (sucrose, mannitol and glucose) and longer treatment periods (Baenas et al., 2014b; Guo, Yuan, \& Wang, 2011a; Guo et al., 2011b). In agreement with our study, the most active GLs inducer among the tested sugars was sucrose (Guo et al., 2011a).

In the present study, total phenolic and total flavonoid contents were significantly affected in a concentration dependent fashion by mannitol, sucrose, $\mathrm{NaCl}, \mathrm{ACC}$ and SA treatment. These observations are in agreement with the increase in phenolics observed in broccoli (Guo, Yuan, \& Wang, 2013), buckwheat (Lim, Park, Kim, Jeong, \& Kim, 2012) and radish sprouts (Yuan et al., 2010) after highconcentration $\mathrm{NaCl}$ treatment. In agreement with our observation, Perez-Balibrea et al. demonstrated an increase of phenolic compounds in broccoli sprouts treated with SA (Pérez-Balibrea et al., 2011) and a similar increase was also observed after low MeJa concentration (10 and $25 \mu \mathrm{M}$ ) treatment (Pérez-Balibrea et al., 2011). On the other hand, the total phenolic compound concentration has been reported to be insensitive to MeJa treatment in broccoli florets (Ku, Choi, Kushad, Jeffery, \& Juvik, 2013). Finally, an increase in total phenolic content in Brassica sprouts after sugars (glucose and sucrose) treatment has been reported (Guo et al., 2011a).

We detected a statistically significant increase of total anthocyanins only after sucrose (both concentration) and MeJa $(30 \mu \mathrm{M})$ treatments. Previous studies showed that exogenous application of MeJa, SA, glucose and sucrose could induce a total anthocyanin increase in radish sprouts (Baenas, Ferreres, GarcíaViguera, \& Moreno, 2015) at longer treatment periods (5 days of treatment) and at different elicitor concentrations. $\mathrm{NaCl}$ treatment has been shown to induce different responses in broccoli sprouts, depending on cultivars and applied concentration (Guo et al., 2013). Finally, it is well established that sugars are strong inducers of anthocyanin biosynthesis in a wide set of plant species (Solfanelli, Poggi, Loreti, Alpi, \& Perata, 2006) and in broccoli sprouts (Guo et al., 2011a,b). Among sugars, sucrose has been shown to be the most effective inducer (Guo et al., 2011a) by a mechanism that has been elucidated in Arabidopsis seedlings (Solfanelli et al., 2006).

In our study, the content of ascorbic acid was significantly increased only by sucrose (both concentrations); while, mannitol induced an increase that did not reach statistical significance. Our data are in agreement with those of Guo (Guo et al., 2011b) that observed an increase of ascorbic acid content in broccoli sprouts after sucrose and mannitol treatment and with those of Nishikawa who observed a sucrose-induced ascorbic acid increase in broccoli florets (Nishikawa et al., 2005).

SA treatment has been shown to cause an increase of vitamin C levels in broccoli sprouts, after 5 to 7 days of treatment (PérezBalibrea et al., 2011). In agreement with our results, PérezBalibrea and coworkers reported that Meja treatment did not affect ascorbic acid concentration in 5 day old broccoli sprouts (PérezBalibrea et al., 2011). On the contrary, $\mathrm{NaCl}$ treatment has been reported to decrease ascorbic acid level in a concentration dependent way (Guo et al., 2013). Even though a similar trend was observed in our study, changes did not reach a statistical threshold.

Usually, biofortification of carotenoids and tocopherols in plants is obtained through genetic modification approaches. However, biotic and abiotic stresses can also affect the level of these compounds in vegetables, and constitute a promising approach. It has been observed, for example, that ozone fumigation increases $\beta$-carotene concentrations in Brassica pekinesis and $\alpha$-tocopherol in B. oleracea var italica (Rozpądek et al., 2015). To our knowledge, this is the first paper reporting on the effect of different elicitors on $\alpha$-tocopherol and $\beta$-carotene levels in broccoli sprouts. We observed that the optimum concentration of ACC, SA and MeJa 
could induce a significant increase of $\beta$-carotene levels, while sucrose and mannitol treatments were associated with a decrease of its content. Previous studies reported that post-harvest exogenous treatment with MeJa stimulates $\beta$-carotene synthesis in tomatoes (Saniewski \& Czapski, 1983) and apples (Pérez, Sanz, Richardson, \& Olías, 1993) and similar results have been obtained using ethylene or SA in citrus (Huang, Liu, Lu, \& Xia, 2008). While, $\mathrm{NaCl}$ treatment has been reported to induce an increase of carotenoid levels in buckwheat sprouts, but at least 5 days of application were required (Lim et al., 2012).

To the best of our knowledge, only one study investigated the effect of elicitor treatments on $\alpha$-tocopherol levels in plants (Saini, Harish Prashanth, Shetty, \& Giridhar, 2014). In this study a concentration-dependent increase of $\alpha$-tocopherol was observed in Moringa oleifera leaves treated with SA and MeJa. In our study, SA treatment induced a concentration-dependent increase (even if this change was not statistically significant), whereas MeJa and sucrose induced a significant reduction (only at the highest concentration) and a significant induction (only at the lowest concentration), respectively.

The ferric reduction power assay (FRAP) and Trolox equivalent antioxidant capacity (TEAC) were used to evaluate the total antioxidant capacity of broccoli sprouts. We observed that mannitol and mannitol/SA were able to induce a significant increase in TEAC and FRAP of broccoli sprouts, respectively. Differently from our results, Guo et al. (2011a,b) observed an increase in FRAP values after sucrose and mannitol treatments: this difference could be due to the older age of broccoli sprouts used in their study ( 7 day-old vs 5 day-old seedlings), as, with ageing the antioxidant capacity of broccoli sprouts drastically decreased (from 3 to 5 days there was a $-40 \%$ and $-45 \%$ reduction for FRAP and TEAC activity, respectively) (data not shown). The same group observed an increase of antioxidant capacity of broccoli sprouts grown in presence of high $\mathrm{NaCl}$ concentrations $(160 \mathrm{mM})$, but low concentrations (up to $80 \mathrm{mM}$ ) had the opposite effect (Guo et al., 2013). Similar results have been also obtained in radish sprouts (Yuan et al., 2010). According to these results we observed that 10 and $50 \mathrm{mM} \mathrm{NaCl}$ induced a significant decrease in the antioxidant capacity measured as FRAP. Similarly, we observed a significant decrease in FRAP values after $30 \mu \mathrm{M}$ MeJa treatment; these results are consistent with those of Barrientos Carvacho et al. (Barrientos Carvacho, Pérez, Zúñiga, \& Mahn, 2014), who observed a Mejainduced decrease of antioxidant capacity (measured using DPPH) in broccoli sprouts. FRAP and TEAC results were highly correlated with each other $(r=0.958)$ and rather correlated to total flavonoids ( $r=0.678$ and 0.649 , respectively) (data not shown). Similar results have already been obtained using different $B$. oleracea varieties of sprouts (Vale, Cidade, Pinto, \& Oliveira, 2014).

PCA identifies sucrose as the most effective eliciting factor to improve the phytochemical composition of broccoli sprouts. In fact, sucrose treatment induced the increase of GLs, total polyphenols, total flavonoids, anthocyanins and vitamin $C$ content, while its effect on vitamin $\mathrm{E}$ was neutral or inductive, according to its concentration. Only, $\beta$-carotene was negatively affected by sucrose treatment; however, the elicitors able to induce $\beta$-carotene level (ACC, SA and MeJa) were not so effective in inducing the expression of other bioactive molecules.

\section{Conclusion}

Our results show that sucrose elicitation is an effective elicitation way to enrich broccoli sprouts of health-promoting bioactive molecules and, then, to possibly enhance their nutritional quality. Further studies are needed to evaluate: (1) if bioactive molecule accumulation really determines an improvement of the functional/biological properties of these bioactive molecule enriched-sprouts and (2) if the induced metabolic changes can affect consumer choices and acceptability.

\section{Declaration of interest}

The authors report no conflicts of interest. The authors alone are responsible for the content and writing of the article.

\section{Acknowledgements}

This work was supported by Italian Ministry of Agriculture, Food \& Forestry (MiPAAF) grants "NUTRIGEA" (DM 30281 23/12/2009).

Kariklia Pascucci is acknowledged for her kind support in the daily lab work.

\section{References}

Abdel-Aal, E. S. M., \& Hucl, P. (1999). A rapid method for quantifying total anthocyanins in blue aleurone and purple pericarp wheats. Cereal Chemistry, 76 (3), 350-354.

Baenas, N., Ferreres, F., García-Viguera, C., \& Moreno, D. A. (2015). Radish sproutsCharacterization and elicitation of novel varieties rich in anthocyanins. Food Research International, 69, 305-312. http://dx.doi.org/10.1016 j.foodres.2015.01.009.

Baenas, N., García-Viguera, C., \& Moreno, D. (2014a). Elicitation: A tool for enriching the bioactive composition of foods. Molecules, 19(9), 13541-13563. http://dx. doi.org/10.3390/molecules190913541.

Baenas, N., García-Viguera, C., \& Moreno, D. A. (2014b). Biotic elicitors effectively increase the glucosinolates content in Brassicaceae sprouts. Journal of Agricultural and Food Chemistry, 62(8), 1881-1889. http://dx.doi.org/10.1021/ jf404876z.

Barrientos Carvacho, H., Pérez, C., Zúñiga, G., \& Mahn, A. (2014). Effect of methyl jasmonate, sodium selenate and chitosan as exogenous elicitors on the phenolic compounds profile of broccoli sprouts. Journal of the Science of Food and Agriculture, 94(12), 2555-2561. http://dx.doi.org/10.1002/jsfa.6596.

Benzie, I. F., \& Strain, J. J. (1996). The ferric reducing ability of plasma (FRAP) as a measure of "antioxidant power": The FRAP assay. Analytical Biochemistry, 239 (1), 70-76. http://dx.doi.org/10.1006/abio.1996.0292.

Björkman, M., Klingen, I., Birch, A. N. E., Bones, A. M., Bruce, T. J. A., Johansen, T. J., \& Stewart, D. (2011). Phytochemicals of Brassicaceae in plant protection and human health - Influences of climate, environment and agronomic practice. $\begin{array}{lll}\text { Phytochemistry, } & \text { 72(7), 538-556. http://dx.doi.org/10.1016/ }\end{array}$ j.phytochem.2011.01.014.

Bodnaryk, R. P. (1994). Potent effect of jasmonates on indole glucosinolates in oilseed rape and mustard. Phytochemistry, 35(2), 301-305. http://dx.doi.org/ 10.1016/S0031-9422(00)94752-6.

Cornelis, M. C., El-Sohemy, A., \& Campos, H. (2007). GSTT1 genotype modifies the association between cruciferous vegetable intake and the risk of myocardial infarction. American Journal of Clinical Nutrition, 86(3), 752-758.

Dewanto, V., Wu, X., Adom, K. K., \& Liu, R. H. (2002). Thermal Processing Enhances the Nutritional Value of Tomatoes by Increasing Total Antioxidant Activity. Journal of Agricultural and Food Chemistry, 50(10), 3010-3014. http://dx.doi.org/ $10.1021 /$ jf0115589.

Fahey, J. W., Zhang, Y., \& Talalay, P. (1997). Broccoli sprouts: An exceptionally rich source of inducers of enzymes that protect against chemical carcinogens. Proceedings of the National Academy of Sciences, 94(19), 10367-10372. http://dx. doi.org/10.1073/pnas.94.19.10367.

Guo, L., Yang, R., Wang Z., Guo, Q. \& Gu, Z. (2014). Effect of $\mathrm{NaCl}$ stress on healthpromoting compounds and antioxidant activity in the sprouts of three broccoli cultivars. International Journal of Food Sciences and Nutrition, 65(4), 476-481. http://dx.doi.org/10.3109/09637486.2013.860583.

Guo, R., Yuan, G., \& Wang, Q. (2011a). Effect of sucrose and mannitol on the accumulation of health-promoting compounds and the activity of metabolic enzymes in broccoli sprouts. Scientia Horticulturae, 128(3), 159-165. http://dx doi.org/10.1016/j.scienta.2011.01.014.

Guo, R., Yuan, G., \& Wang, Q. (2011b). Sucrose enhances the accumulation of anthocyanins and glucosinolates in broccoli sprouts. Food Chemistry, 129(3), 1080-1087. http://dx.doi.org/10.1016/j.foodchem.2011.05.078.

Guo, R., Yuan, G., \& Wang, Q. (2013). Effect of $\mathrm{NaCl}$ treatments on glucosinolate metabolism in broccoli sprouts. Journal of Zhejiang University Science B, 14(2), 124-131. http://dx.doi.org/10.1631/jzus.B1200096.

Huang, R.-H., Liu, J.-H., Lu, Y.-M., \& Xia, R.-X. (2008). Effect of salicylic acid on the antioxidant system in the pulp of "Cara cara" navel orange (Citrus sinensis L Osbeck) at different storage temperatures. Postharvest Biology and Technology, 47(2), 168-175. http://dx.doi.org/10.1016/j.postharvbio.2007.06.018.

Kapasakalidis, P. G., Rastall, R. A., \& Gordon, M. H. (2006). Extraction of polyphenols from processed black currant (Ribes nigrum L.) residues. Journal of Agricultural and Food Chemistry, 54(11), 4016-4021. http://dx.doi.org/10.1021/jf0529991. 
Ku, K. M., Choi, J. H., Kushad, M. M., Jeffery, E. H., \& Juvik, J. A. (2013). Pre-harvest methyl jasmonate treatment enhances cauliflower chemoprotective attributes without a loss in postharvest quality. Plant Foods for Human Nutrition, 68(2), 113-117. http://dx.doi.org/10.1007/s11130-013-0356-y.

Ku, K. M., Jeffery, E. H., \& Juvik, J. A. (2014). Optimization of methyl jasmonate application to broccoli florets to enhance health-promoting phytochemical content. Journal of the Science of Food and Agriculture, 94(10), 2090-2096. http:// dx.doi.org/10.1002/jsfa.6529.

Lim, J. H., Park, K. J., Kim, B. K., Jeong, J. W., \& Kim, H. J. (2012). Effect of salinity stress on phenolic compounds and carotenoids in buckwheat (Fagopyrum esculentum M.) sprout. Food Chemistry, 135(3), 1065-1070. http://dx.doi.org/10.1016/ j.foodchem.2012.05.068.

Liu, X., \& Lv, K. (2013). Cruciferous vegetables intake is inversely associated with risk of breast cancer: A meta-analysis. Breast (Edinburgh, Scotland), 22(3), 309-313. http://dx.doi.org/10.1016/j.breast.2012.07.013.

López-Berenguer, C., Martínez-Ballesta, M. C., García-Viguera, C., \& Carvajal, M. (2008). Leaf water balance mediated by aquaporins under salt stress and associated glucosinolate synthesis in broccoli. Plant Science, 174(3), 321-328. http://dx.doi.org/10.1016/j.plantsci.2007.11.012.

Maiani, G., Pappalardo, G., Ferro-Luzzi, A., Raguzzini, A., Azzini, E., Guadalaxara, A., \& Mobarhan, S. (1995). Accumulation of $\beta$-carotene in normal colorectal mucosa and colonic neoplastic lesions in humans. Nutrition and Cancer, 24(1), 23-31.

Maldini, M., Baima, S., Morelli, G., Scaccini, C., \& Natella, F. (2012). A liquid chromatography-mass spectrometry approach to study "glucosinoloma" in broccoli sprouts. Journal of Mass Spectrometry, 47(9), 1198-1206. http://dx.doi. org/10.1002/jms.3028.

Margolis, S. A., Paule, R. C., \& Ziegler, R. G. (1990). Ascorbic and dehydroascorbic acids measured in plasma preserved with dithiothreitol or metaphosphoric acid. Clinical Chemistry, 36(10), 1750-1755.

Mikkelsen, M. D., Petersen, B. L., Glawischnig, E., Jensen, A. B., Andreasson, E., \& Halkier, B. A. (2003). Modulation of CYP79 genes and glucosinolate profiles in Arabidopsis by defense signaling pathways. Plant Physiology, 131(1), 298-308. http://dx.doi.org/10.1104/pp.011015.

Nishikawa, F., Kato, M., Hyodo, H., Ikoma, Y., Sugiura, M., \& Yano, M. (2005). Effect of sucrose on ascorbate level and expression of genes involved in the ascorbate biosynthesis and recycling pathway in harvested broccoli florets. Journal of Experimental Botany, 56(409), 65-72. http://dx.doi.org/10.1093/jxb/eri007.

Pellegrini, N., Serafini, M., Colombi, B., Del Rio, D., Salvatore, S., Bianchi, M., \& Brighenti, F. (2003). Total antioxidant capacity of plant foods, beverages and oils consumed in Italy assessed by three different in vitro assays. Journal of Nutrition, 133(9), 2812-2819.

Pérez, A. G., Sanz, C., Richardson, D. G., \& Olías, J. M. (1993). Methyl jasmonate vapor promotes $\beta$-carotene synthesis and chlorophyll degradation in Golden Delicious apple peel. Journal of Plant Growth Regulation, 12(3), 163-167. http://dx.doi.org/ 10.1007/BF00189648.

Pérez-Balibrea, S., Moreno, D. A., \& García-Viguera, C. (2011). Improving the phytochemical composition of broccoli sprouts by elicitation. Food Chemistry, 129(1), 35-44. http://dx.doi.org/10.1016/j.foodchem.2011.03.049.

Plumb, G. W., Lambert, N., Chambers, S. J., Wanigatunga, S., Heaney, R. K., Plumb, J. A., \& Williamson, G. (1996). Are whole extracts and purified glucosinolates from cruciferous vegetables antioxidants? Free Radical Research, 25(1), 75-86.

Rozpądek, P., Nosek, M., Ślesak, I., Kunicki, E., Dziurka, M., \& Miszalski, Z. (2015). Ozone fumigation increases the abundance of nutrients in Brassica vegetables: broccoli (Brassica oleracea var. italica) and Chinese cabbage (Brassica pekinensis). European Food Research and Technology, 240(2), 459-462. http:// dx.doi.org/10.1007/s00217-014-2372-z.

Saini, R. K., Harish Prashanth, K. V., Shetty, N. P., \& Giridhar, P. (2014). Elicitors, SA and $\mathrm{MJ}$ enhance carotenoids and tocopherol biosynthesis and expression of antioxidant related genes in Moringa oleifera Lam. leaves. Acta Physiologiae Plantarum, 36(10), 2695-2704. http://dx.doi.org/10.1007/s11738-014-1640-7.

Saniewski, M., \& Czapski, J. (1983). The effect of methyl jasmonate on lycopene and $\beta$-carotene accumulation in ripening red tomatoes. Experientia, 39(12), 1373-1374. http://dx.doi.org/10.1007/BF01990110.

Sharpless, K. E., Arce-Usuna, M., Thomas, J. B., \& Gill, L. M. (1999). Value assignment of retinol, retinyl palmitate, tocopherol, and carotenoid concentrations in standard reference material 2383 (baby food composite). Journal of AOAC International, 82(2), 288-296.

Singh, J., Upadhyay, A. K., Prasad, K., Bahadur, A., \& Rai, M. (2007). Variability of carotenes, vitamin C, E and phenolics in Brassica vegetables. Journal of Food Composition and Analysis, 20(2), 106-112. http://dx.doi.org/10.1016/j. jfca.2006.08.002.

Singleton, V. L., Orthofer, R., \& Lamuela-Raventós, R. M. (1999). Oxidants and Antioxidants Part A. Methods in Enzymology (vol. 299). http://dx.doi.org/10.1016/ S0076-6879(99)99017-1.

Solfanelli, C., Poggi, A., Loreti, E., Alpi, A., \& Perata, P. (2006). Sucrose-specific induction of the anthocyanin biosynthetic pathway in Arabidopsis. Plant Physiology, 140(2), 637-646. http://dx.doi.org/10.1104/pp.105.072579.

Vale, A. P., Cidade, H., Pinto, M., \& Oliveira, M. B. P. P. (2014). Effect of sprouting and light cycle on antioxidant activity of Brassica oleracea varieties. Food Chemistry, 165, 379-387. http://dx.doi.org/10.1016/j.foodchem.2014.05.122.

Yuan, G., Wang, X., Guo, R., \& Wang, Q. (2010). Effect of salt stress on phenolic compounds, glucosinolates, myrosinase and antioxidant activity in radish sprouts. Food Chemistry, 121(4), 1014-1019. http://dx.doi.org/10.1016/ j.foodchem.2010.01.040. 